\title{
Clipopexy: An Anchoring Technique to Avoid Compression of Adjacent Neurovascular Structures by Aneurysm Clip: Report of Two Cases
}

\author{
Martin M. Mortazavi ${ }^{1}$, Ali Ravanpay ${ }^{1}$, Laligam N. Sekhar $^{1}$ \\ 1. Department of Neurosurgery, University of Washington School of Medicine, Seattle, WA
}

$\square$ Corresponding author: Ali Ravanpay, ravanpay@u.washington.edu

Disclosures can be found in Additional Information at the end of the article

\section{Abstract}

Rupture of an intracranial aneurysm is a serious neuro-vascular event requiring immediate management to avoid devastating neurological sequelae. Despite the rapid evolution of endovascular techniques, clipping is still a necessary tool in aneurysms with non-optimal dome-to-neck ratio and in younger patients. Occasionally, optimal positioning of an aneurysm clip with optimal obliteration of the aneurysm is not possible without compression of adjacent neurovascular structures. In these cases, the compressed neurovascular structure can be decompressed while maintaining the position of the clip on the aneurysm through an anchoring technique. Here, we describe this technique, in which the aneurysm clip is pulled away from the compressed neurovascular structures by sutures.

Categories: Neurosurgery

Keywords: aneurysm clip, subarachnoid hemorrhage, clipopexy

\section{Introduction}

The decision on how to clip a cerebral aneurysm is complex. It is influenced by the size, form, location, and anatomical relationship to adjacent neurovascular structures [1-2]. Three key considerations during clip placement are: 1) optimal placement at the neck of the aneurysm to stop the flow into, and thus obliterate the aneurysm, 2) to prevent residual aneurysm neck proximal to the aneurysm clip, and 3) to avoid immediate or delayed compressional injury to adjacent neurovascular structures by the aneurysm clip.

Received 03/02/2014 Review began 03/02/2014 Review ended 03/31/2014 Published 03/31/2014

\section{C) Copyright 2014}

Mortazavi et al. This is an open access article distributed under the terms of the Creative Commons Attribution License CC-BY 3.0., which permits unrestricted use, distribution, and reproduction in any medium, provided the original author and source are credited.
Aneurysms are often in locations surrounded by multiple eloquent structures, such as the optic nerve, the occulomotor nerve, and the cerebral arteries and their branches. An optimal obliteration of an aneurysm by a clip alone is not sufficient, as care must be taken to avoid compression on the adjacent nerves and arteries. A review of literature reveals reports of unfortunate neurologic injury secondary to clip misplacement or movement [3]. Repositioning of the clip can often maintain a good obliteration without a residual neck and compression of adjacent neurovascular structures. However, in certain cases, when repositioning of the aneurysm clip would result in a neck residual, an optimal obliteration of an aneurysm is achieved at the cost of slight compression of an adjacent nerve or artery by the aneurysm clip. In these cases, clipopexy can be used. In this technique, one or two sutures through the aneurysm clip, and anchored to the adjacent dura, hold the clip away from the compressed nerve or artery. The senior author (LNS) has used this technique in more than 20 cases (out of approximately 500 aneurysm operations involving the ICA). We usually use a 7.0 Prolene. Here 
within, we describe two illustrative cases.

\section{Technical Report}

\section{Technical report 1}

An 87-year-old woman was admitted for subarachnoid hemorrhage (SAH). She was Hunt-Hess Grade 2, Fisher Grade 3, and had hydrocephalus (Figure 1A). CT angiography (CTA) and a subsequent cerebral catheter angiogram confirmed a left-sided 12 x $5 \mathrm{~mm}$ posterior communicating aneurysm (Figure $1 B, 1 C$ ). Due to its complex shape, the aneurysm was deemed unsuitable for endovascular treatment.

After obtaining informed consent from patient's legal next of kin, a left fronto-temporal craniotomy with posterolateral orbitotomy was performed. The optic nerve was decompressed extradurally, and the anterior clinoid process was removed. The Sylvian fissure was opened in a lateral to medial fashion, and the optico-carotid cistern was opened to release CSF. The aneurysm was projecting posteriorly and had a broad neck. Due to severe arteriosclerosis and calcification with high risk of distal embolus, no temporary clamping was performed; however, the blood pressure was temporarily lowered to $90 \mathrm{~mm} \mathrm{Hg}$ systolic during the aneurysm dissection and clipping. The anterior choroidal artery was firmly adherent to the lateral surface of the aneurysm and was sharply dissected off the aneurysm. A fenestrated 45degree angle clip (Sugita Mizuho Titanium, Tokyo, Japan) was utilized and carefully worked between the aneurysm neck and the anterior choroidal artery. The aneurysm collapsed after the clip placement. However, the clip compressed the origin of the ipsilateral A1 artery. Repositioning of the aneurysm clip with the tightly adherent anterior choroidal artery on top of it was not deemed suitable. In order to release the tension, the A1-artery was dissected distally to the anterior communicating artery, but the compression remained. As no other option remained, we chose to tilt the aneurysm clip off the A1-artery by suturing the clip to dura near the anterior clinoid process by using a 7.0 Prolene suture (Figure 2). An Indocyanine Green (ICG) angiogram showed all the vessels to be patent and non-compressed. The dura mater was closed in a watertight fashion. Remaining closure was performed in the usual fashion, in layers.

A postoperative CT scan demonstrated normal postoperative changes without any sign of infarct (Figure 2A). An MRI confirmed lack of any strokes (Figure 2B). Postoperative cerebral catheter angiogram revealed complete obliteration of the aneurysm and no compression of the A1-artery (Figure 2C). The patient recovered well from the operation and is currently progressing towards rehabilitation. 


\section{Cureus}
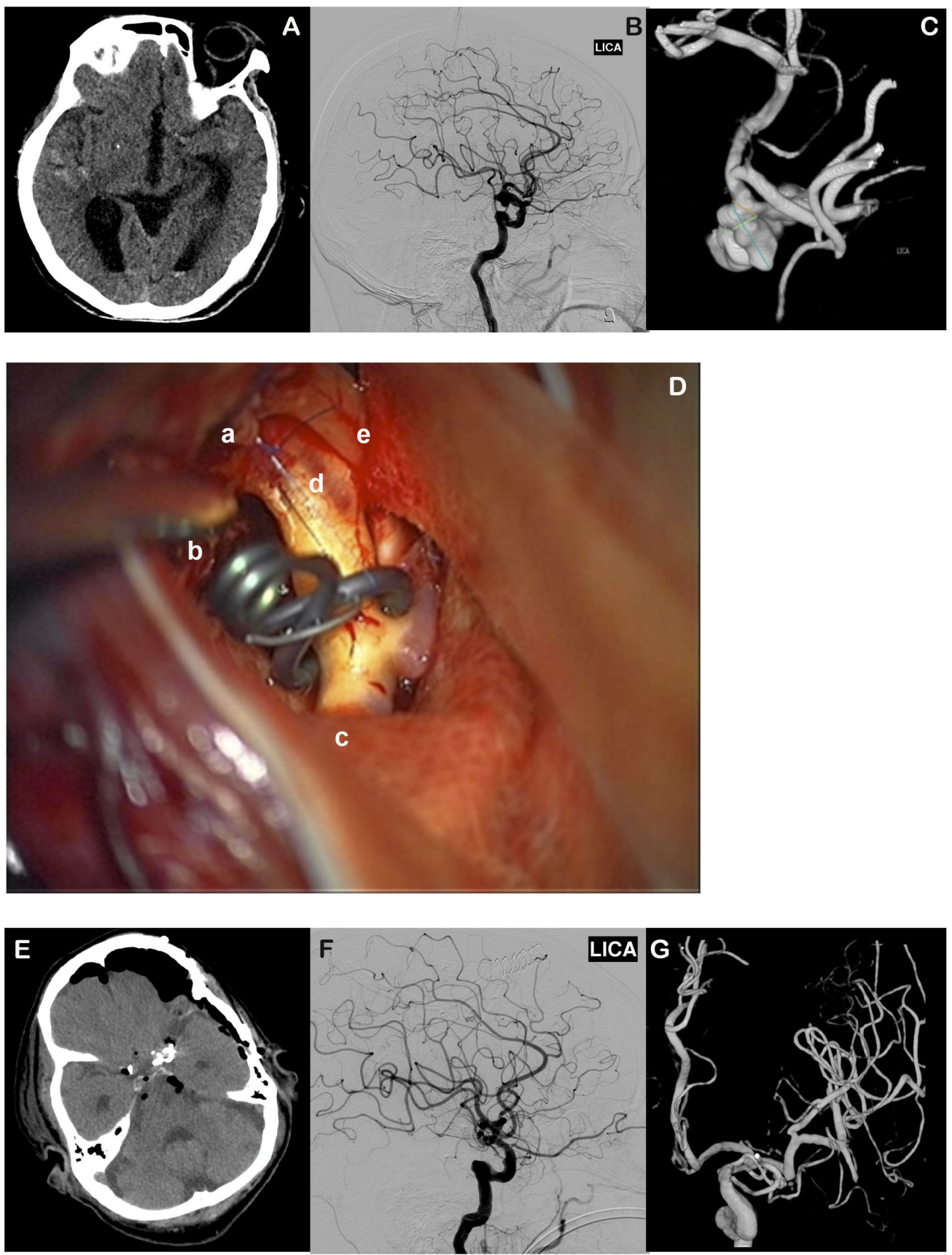

\section{FIGURE 1: Pre- and postoperative imaging and clipopexy;}

\section{Technical Report 1.}

Panel A: CT of the brain upon arrival at our hospital demonstrates bilateral Sylvian fissure, subarachnoid hemorrhage, and intraventricular hemorrhage. Panel B: DSA, Left Internal Carotid Injection (LICA), lateral view, demonstrating a complex supraclinoid aneurysm. Panel C: DSA, $3 \mathrm{D}$ reconfiguration demonstrates $12 \times 5 \mathrm{~mm}$ bilobed aneurysm arising from the left internal carotid artery with $4.6 \mathrm{~mm}$ neck. Panel $\mathrm{D}$ : Anchoring the curve of the microclip against the nearby ipsilateral anterior clinoid dura to lift the clip anterior and away from the posterior anterior cerebral artery branching point (a: 7.0 Prolene suture, b: microclip, c: M1, d: ICA, e: Optic nerve) Panel E: Postoperative CT scan demonstrating aneurysm clip, postoperative pneumocephalus, and no new hemorrhage. Panel F: Postoperative DSA, LICA injection, lateral view demonstrating obliteration of previous aneurysm. Panel G: Postoperative DSA, 3D reconfiguration demonstrates successful clipping of aneurysm with no residual neck. 


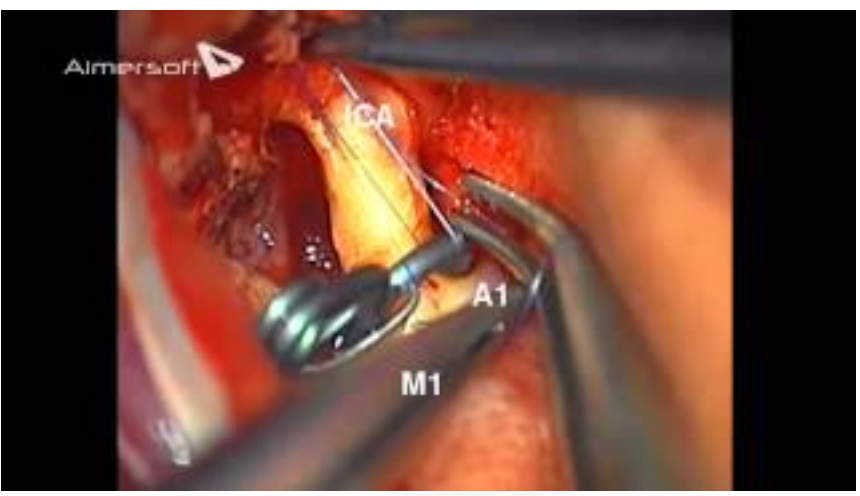

\section{VIDEO 1: Clipopexy Technical Report \#1}

Edited video segment demonstrating highlights of the clipopexy in a representative case with a left-sided $12 \times 5 \mathrm{~mm}$ posterior communicating aneurysm.

View video here: http://www.youtube.com/watch?v=z1oAh3aBhjl

\section{Technical report 2}

A 59-year-old man was found to have an asymptomatic unruptured aneurysm of the basilar tip during the trauma work-up after an automobile accident. The aneurysm measured at $8 \times 7 \mathrm{~mm}$ with a complex and wide neck $(5 \mathrm{~mm})$. There was an additional outpouching of the anteroinferior aspect of the aneurysm measuring $1 \mathrm{~mm}$ with incorporation of the origins of both P1 segments into the neck of the aneurysm. Due to its complex architecture, endovascular treatment was not felt to be optimal for this aneurysm.

As the aneurysm was above the floor of the sella, a left-sided fronto-temporal craniotomy with orbito-zygomatic osteotomy was performed after obtaining informed consent. The superior orbital fissure and the optic nerve were decompressed extradurally. The Sylvian fissure was opened in the lateral to medial fashion. In order to clear the trajectory to the aneurysm, anterior clinoidectomy, as well as sectioning of the ipsilateral non-dominant posterior communicating artery, was performed (If the ipsilateral posterior communicating artery would have been fetal, we would have favored a subtemporal approach). Subsequently, the proximal portion of the internal carotid artery was completely mobilized. The aneurysm was exposed through the opticocarotid triangle. Despite this maneuver, proximal control could not be achieved. Therefore, the posterior clinoid process was resected. The patient was placed in burst suppression, and a temporary clip was placed on the basilar artery proximal to the superior cerebellar artery, carefully avoiding perforating vessels in this area. The aneurysm was subsequently dissected with its complex neck exposed. The left posterior cerebral artery was partially arising from the neck of the aneurysm. An $11 \mathrm{~mm}$ long straight clip (FT760T, Yasargil Titanium, Aesculap, Center Valley, USA) was placed across the main body of the aneurysm in the antero-posterior direction, angling more posteriorly because of the overflowing neck at the back of the aneurysm. Unfortunately, this left a fairly prominent portion of the aneurysmal neck unsecured anteriorly. The posterior clip had to be moved slightly, and an additional mini clip (FT760T) was placed slightly angled, and in a direction that was parallel to the P1 vessels, across the proximal portion of the neck of the aneurysm. Optimal flow was verified with micro Doppler and Indocyanine green (ICG) angiogram.

When the clips were inspected, it was apparent that the macro clip was compressing the left optic nerve at its junction with the optic tract. Despite opening the falciform ligament, the compression persisted. Repositioning of the aneurysm clips to a better position was not possible. Clipopexy of this clip was performed by suturing it towards the paraclinoidal dura, which completely decompressed the optic nerve. 


\section{Cureus}

The patient did very well postoperatively. At one year follow-up, he had no neurologic deficits and no evidence of residual recurrence of the clipped aneurysm.
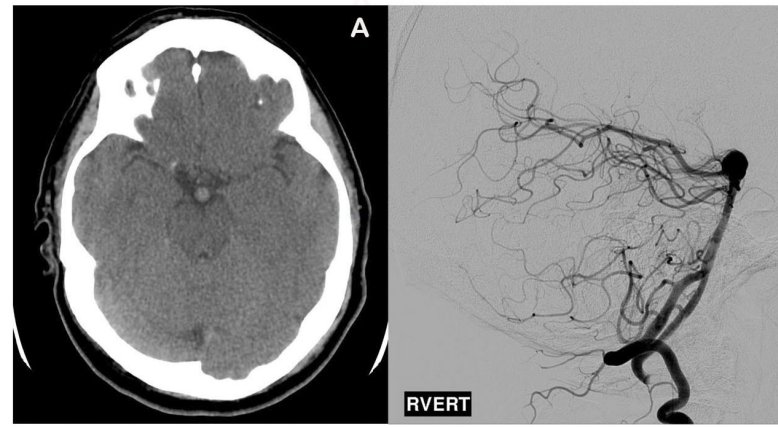

B
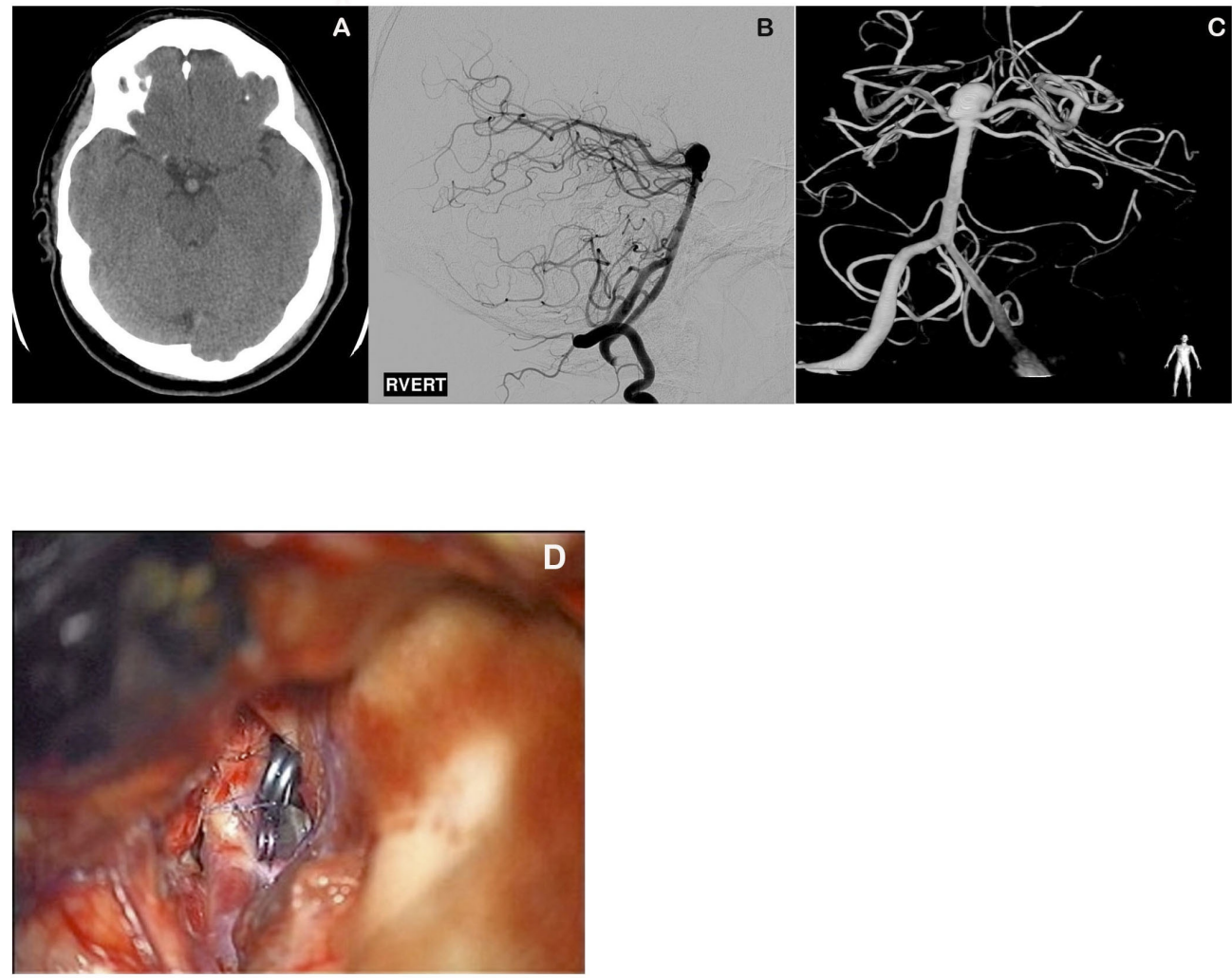

D
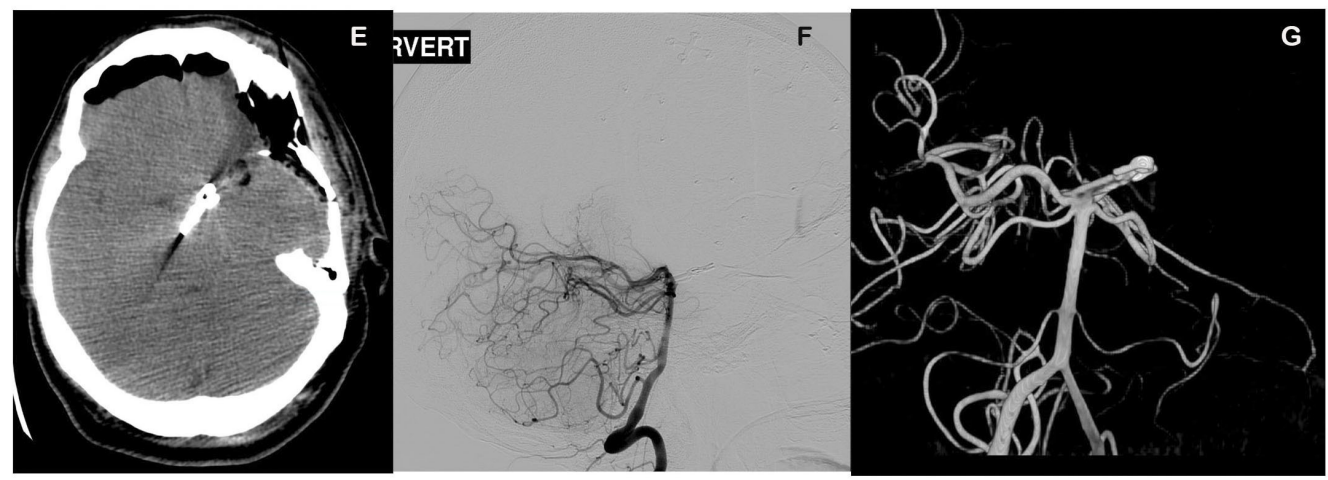

FIGURE 2: Pre- and postoperative imaging and clipopexy;

\section{Technical Report 2}

Panel A: CT of the brain as part of trauma work-up after an automobile accident without evidence of subarachnoid hemorrhage. Panel B: DSA, right vertebral artery injection, lateral view, demonstrating a complex basilar tip aneurysm. Panel C: DSA, 3D reconfiguration demonstrates a broad base $8.2 \times 6.7 \times 8.1 \mathrm{~mm}$ complex aneurysm arising from the basilar artery encompassing the root of bilateral P1 segments. Panel D: Anchoring the microclip against the nearby ipsilateral clinoid dura to lift the clip away from the optic nerve Panel E: Postoperative CT scan demonstrating aneurysm clip, postoperative pneumocephalus and no new hemorrhage. Panel F: Postoperative DSA, right vertebral artery injection, lateral view demonstrating obliteration of previous aneurysm. Panel G: Postoperative DSA, 3D reconfiguration demonstrates successful clipping of aneurysm with no residual neck. 


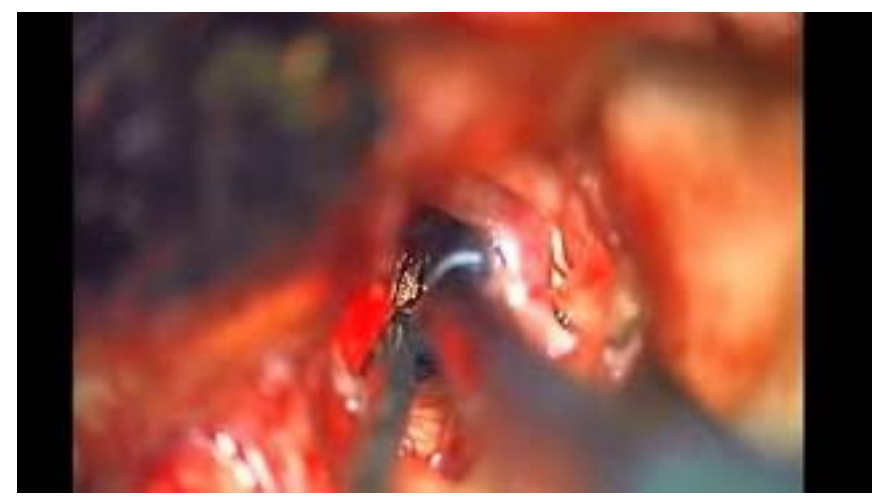

\section{VIDEO 2: Clipopexy Technical Report \#2}

Edited video segment demonstrating highlights of the clipopexy in a second representative case with an asymptomatic unruptured aneurysm of the basilar tip measuring $8 \times 7 \mathrm{~mm}$ with a complex and wide neck $(5 \mathrm{~mm})$.

View video here: http://www.youtube.com/watch?v=8SMD8HqQOOk

\section{Discussion}

To clip an aneurysm successfully without injuring adjacent structures requires expert planning and delicate microvascular choreography. Occasionally, despite all efforts and due to anatomical constraints, part of the aneurysm clip compresses adjacent neurovascular structures. Lifting of one structure off another through a suture is called pexy.

In this report, we introduce a technique where the aneurysm clip, while in perfect position for obliterating the aneurysm, is tilted away from the adjacent compressed structure, being an artery or nerve, in order to avoid immediate or delayed injury. We call this technique Clipopexy. The term pexy is used in other contexts. Microvascular decompression for trigeminal neuralgia or hemifacial spasm is a well-known procedure. In this technique, the decompression off of the cranial nerves is achieved through a pledget. Sometimes placement of pledgets alone is not enough, and the compressing artery can be sutured off of the cranial nerve to the adjacent dura. This is called an arteriopexy. The senior author has applied this technique in more than 20 cases throughout his career. Mostly, it has been used in basilar and vertebral compression syndromes, compressing pons and medulla oblongata, respectively. One or two 7.0 Prolone sutures are used to hold the artery off of the pons and medulla, respectively, and pulling them off towards the adjacent dura. Review of the literature reveals references to this form of pexy in treatment of cerebral vascular disease by the senior author (LNS) as a contributor to "Samii's Essentials in Neurosurgery" (Ramina, et al., 2008, Chapter 32 "Bypass and Vascular Reconstruction for Anterior Circulation Aneurysms", pp 329-351) and by Nussbaum in "Video Atlas of Intracranial Aneurysm Surgery" (Nussbaum, 2012, Chapter 3 "Paraclinoid Aneurysms," pp 25-35). Here, a modification of the pexy-technique is used in which suturing the site of the graft to the proximal vessel relieves a kink in the proximal anastomosis site.

Further evolution of the pexy-technique is Clipopexy. In this technique, the same principle is used to tilt an aneurysm clip off of eloquent neurovascular structures.

In our illustrative Case 1 presented here, due to limitations that the calcified parental vessel and the tightly adherent anterior choroidal artery provided, with no other alternative clip position possible, we were able to maintain full obliteration of the aneurysm without any residual neck and avoid mechanical compression of the A1 segment by tilting the aneurysm clip away from the origin of the A1 by two sutures anchored at the adjacent dura. A follow-up MRI 
confirmed intact flow and absence of stroke in the ACA territory, confirming the functional application of this technique. Similarly, in Case 2, the perfect obliteration of the complex basilar tip aneurysm would have been sacrificed by repositioning of the larger clip in order to release the tension off of the optic nerve. Hence, the aneurysm position was maintained, while releasing the optic nerve decompression by this technique. Attention must be given to the trajectory and tension of the pexy sutures to ensure continued safe placement of the aneurysm clip without the risk of traction at the neck of the aneurysm, while pulling the clip off of the point of undesired compression. Depending on the need, one or two sutures can be used. In both illustrated cases, compression of the adjacent neurovascular structures may have increased postoperatively with brain returning to its anatomical position.

\section{Conclusions}

Clipopexy can safely be performed to relieve pressure off of neurovascular structures adjacent to an aneurysm clip by tilting the clip off of these structures through anchoring the aneurysm clip to the adjacent dura with micro-sutures.

\section{Additional Information}

\section{Disclosures}

Human subjects: All authors have confirmed that this study did not involve human participants or tissue. Animal subjects: All authors have confirmed that this study did not involve animal subjects or tissue. Conflicts of interest: In compliance with the ICMJE uniform disclosure form, all authors declare the following: Payment/services info: All authors have declared that no financial support was received from any organization for the submitted work. Financial relationships: All authors have declared that they have no financial relationships at present or within the previous three years with any organizations that might have an interest in the submitted work. Other relationships: All authors have declared that there are no other relationships or activities that could appear to have influenced the submitted work.

\section{Acknowledgements}

The authors would like to thank members of the Harborview Medical Center operating room staff.

\section{References}

1. Wiebers DO, Whisnant JP, Huston J, 3rd, Meissner I, Brown RD, Jr., Piepgras DG, et al.: Unruptured intracranial aneurysms: natural history, clinical outcome, and risks of surgical and endovascular treatment. Lancet. 2003, 362:103-10.

2. Molyneux A, Kerr R, Stratton I, Sandercock P, Clarke M, Shrimpton J, Holman R; International Subarachnoid Aneurysm Trial (ISAT) Collaborative Group: International Subarachnoid Aneurysm Trial (ISAT) of neurosurgical clipping versus endovascular coiling in 2143 patients with ruptured intracranial aneurysms: a randomized trial. The Lancet. 2002, 360:1267-74.

3. Zairi F, Thines L, Bourgeois P, Ayachi M, Lejeune JP: Anterior optic pathways compression by a surgical clip mobilized by delayed chronic hydrocephalus after treatment of a ruptured anterior communicating artery aneurysm (in French). Neuro-Chirurgie. 2012, 58:34-6. 10.1016/j.neuchi.2011.07.006

4. Sekhar LN, Natarajan SK, Brtiz G, Ghodke B: Chapter 32: Bypass and Vascular Reconstruction for Anterior Circulation Aneurysms. Samii's Essentials in Neurosurgery. Ramina R., Pires Aguiar PH., Tatagiba M (ed): Springer, New York, NY; 2008. 329-351.

5. Nussbaum ES: Chapter 3: Paraclinoid Aneurysms. Video Atlas of Intracranial Aneurysm Surgery. Nussbaum ES (ed): Thieme Publishers, New York, NY; 2012. 25-35. 